\title{
Globe
}

Revue internationale d'études québécoises

\section{La naissance comme accident providentiel. Le risque et les allocations familiales au début du $\mathrm{XX}^{\mathrm{e}}$ siècle au Québec Childbirth as a Fortuitous Accident. Risk and Family Allowances in Quebec at the Beginning of the Twentieth Century}

\section{Martin Petitclerc}

Volume 16, numéro 2, 2013

L'avenir (probable) du passé : le risque et l'histoire du Québec

URI : https://id.erudit.org/iderudit/1025216ar

DOI : https://doi.org/10.7202/1025216ar

Aller au sommaire du numéro

Éditeur(s)

Globe, Revue internationale d'études québécoises

ISSN

1481-5869 (imprimé)

1923-8231 (numérique)

Découvrir la revue

Citer cet article

Petitclerc, M. (2013). La naissance comme accident providentiel. Le risque et les allocations familiales au début du XX ${ }^{\mathrm{e}}$ siècle au Québec. Globe, 16(2), 119-142. https://doi.org/10.7202/1025216ar
Résumé de l'article

Dans la première moitié $\mathrm{du} \mathrm{xx}^{\mathrm{e}}$ siècle, le développement du savoir assurantiel appliqué aux politiques sociales a permis de repenser les termes de la question sociale. À ce moment, une coalition éclectique de réformateurs, d'intellectuels, de politiciens et de groupes sociaux reformule un large éventail de problèmes sociaux comme des accidents et des risques sociaux, plutôt que le résultat de la faute et de la responsabilité individuelles. Un tel déplacement a permis de contourner plusieurs contraintes du mode de régulation libéral, tout en balisant étroitement les discours et les pratiques de la solidarité au sein de la communauté politique contemporaine. Dans cet article, je me propose d'examiner ces enjeux en me concentrant sur le projet d'allocations familiales de Léon Lebel, un jésuite actif au sein de l'influent mouvement du catholicisme social. Ce projet était très inusité puisqu'il était basé sur une conception matérialiste de la naissance qui en faisait un « accident providentiel » pour le chef de famille, ce qui permettait d'envisager la mutualisation de ce risque par la technologie assurantielle. Bien que ce projet n’ait jamais été appliqué, il s'agit tout de même d'un exemple convaincant de l'importance stratégique du savoir assurantiel et des discours sur le risque - même pour un catholique social comme Lebel - dans les débats sur les politiques sociales pendant une grande partie du $\mathrm{xx}^{\mathrm{e}}$ siècle. 


\title{
LA NAISSANCE COMME ACCIDENT PROVIDENTIEL. LE RISQUE ET LES ALLOCATIONS FAMILIALES AU DÉBUT DU XX ${ }^{E}$ SIÈCLE AU QUÉBEC ${ }^{1}$
}

\author{
MARTIN PETITCLERC \\ UQAM \\ Centre d'histoire des régulations sociales \\ Centre de recherche sur les innovations sociales
}

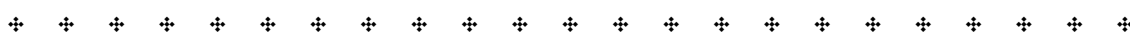

Résumé - Dans la première moitié du XXe siècle, le développement du savoir assurantiel appliqué aux politiques sociales a permis de repenser les termes de la question sociale. À ce moment, une coalition éclectique de réformateurs, d'intellectuels, de politiciens et de groupes sociaux reformule un large éventail de problèmes sociaux comme des accidents et des risques sociaux, plutôt que le résultat de la faute et de la responsabilité individuelles. Un tel déplacement a permis de contourner plusieurs contraintes du mode de régulation libéral, tout en balisant étroitement les discours et les pratiques de la solidarité au sein de la communauté politique contemporaine. Dans cet article, je me propose d'examiner ces enjeux en me concentrant sur le projet d'allocations familiales de Léon Lebel, un jésuite actif au sein de l'influent mouvement du catholicisme social. Ce projet était très inusité puisqu'il était basé sur une conception matérialiste de la naissance qui en faisait un "accident providentiel » pour le chef de famille, ce qui permettait d'envisager la mutualisation de ce risque par la technologie assurantielle.

$$
\div+\div
$$

1. Je tiens à remercier Anne-Marie Buisson et toute l'équipe d'assistants et d'assistantes de recherche du Centre d'histoire des régulations sociales pour l'aide qu'ils m'ont apportée lors de la recherche ayant mené à la rédaction de cet article. Les commentaires des deux évaluateurs anonymes m'ont permis d'améliorer la première version de ce texte. Je remercie également ma collègue Magda Fahrni pour ses commentaires éclairants. Je demeure évidemment seul responsable des propos qui vont suivre. Cette recherche a bénéficié du soutien financier du Fonds de recherche Société et culture du Québec ainsi que de la Fondation canadienne de l'innovation du Canada. 
Bien que ce projet n'ait jamais été appliqué, il s'agit tout de même d'un exemple convaincant de l'importance stratégique du savoir assurantiel et des discours sur le risque - même pour un catholique social comme Lebel - dans les débats sur les politiques sociales pendant une grande partie du XXe siècle.

\section{Childbirth as a fortuitous accident. Risk and family allowances in Quebec at the beginning of the twentieth century}

Abstract - In the first half of the twentieth century, the "social question" was reframed by applying knowledge developed in the field of insurance to social policy. At the time, an eclectic coalition of reformers, intellectuals, politicians and social groups redefined a wide array of social problems in terms of risk and misfortune, rather than viewing them through the prism of personal conduct and responsibility. This shift allowed policymakers to bypass many of the constraints imposed by the nineteenth-century liberal mode of regulation, while shaping practices and discourses of solidarity within the contemporary political community. In this article, I examine these issues by focusing on the family allowances project of Léon Lebel, a Jesuit active in the influential Catholic social movement. This project was highly unusual in that it was based on the materialist notion that childbirth should be considered a "fortuitous accident" for the head of the household, thereby allowing for the risk to be mutualized through insurance practices. Although this particular proposal never became law, it still serves as a persuasive example of the strategic importance of insurance knowledge and discourses on risk in social policy debates during much of the twentieth century, even for a social Catholic like Lebel.

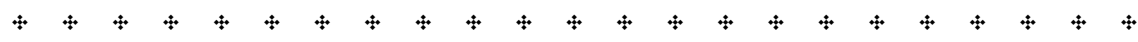

Le risque de famille, si on peut appeler ainsi la venue de l'enfant, est tout à l'avantage de la société.

Léon Lebel, $1928^{2}$

Dans la première moitié du XXe siècle, le développement du savoir assurantiel appliqué aux politiques sociales a permis de repenser les termes de la question sociale. À ce moment, une coalition éclectique de réformateurs, d'intellectuels, de politiciens et de groupes sociaux reformule audacieusement les problèmes sociaux en les présentant comme des «accidents » et des "risques sociaux " plutôt que le résultat de la faute et de la responsabilité individuelles. Un tel déplacement a permis de contourner plusieurs contraintes du mode de régulation libéral, tout en balisant étroitement le ter-

$$
\div \div
$$

2. Léon LEBEL, Pourquoi les allocations familiales? Raisons et objections, Montréal, Arbour et Dumont, 1928 . 
ritoire de la solidarité au sein de la communauté politique contemporaine ${ }^{3}$. Malgré l'importance du développement du savoir assurantiel pour comprendre la genèse de l'État-providence, relativement peu d'historiens québécois et canadiens se sont intéressés à cette question. Dans le cas du Québec, ce savoir est pourtant au cœur des débats sur la politique sociale depuis l'adoption de la loi sur les accidents du travail de 1909. Par la suite, le discours sur le risque est progressivement étendu à une foule de problèmes sociaux, tels que le chômage, la maladie, la vieillesse et, comme nous le verrons, la famille nombreuse. À cet égard, malgré la réputation de conservatisme du Québec en matière sociale avant les années 1960, qui est en bonne partie attribuable à la place centrale occupée par le "personnage de l'indigent " dans le système privé et catholique d'assistance ${ }^{4}$, il est intéressant de constater qu'une partie importante de la société québécoise est déjà acquise aux principes des assurances sociales pour couvrir un éventail assez large de risques sociaux au tournant des années 1930. C'est ce qui ressort notamment des travaux de la Commission sur les assurances sociales du Québec (CASQ) à ce moment ${ }^{5}$.

Dans cet article, je m'intéresserai au projet d'allocations familiales de Léon Lebel, un jésuite actif au sein de l'École sociale populaire (ESP) et aumônier général de l'Union des cultivateurs catholiques ${ }^{6}$ (UCC). Ce projet, qui a donné lieu à la première campagne politique d'allocations familiales au Canada $^{7}$, est fascinant parce qu'il s'appuie explicitement sur le discours de

$$
\div+\div
$$

3. L'étude classique à ce sujet est de François Ewald (François EWALD, L'État providence, Paris, Grasset, 1986). Pour une discussion critique récente sur les enjeux du risque dans la construction de l'Étatprovidence, voir Martin PETITCLERC, "L'État providence, la société du risque et les fondements de la communauté politique ", Martin PetiTClerC et David NigET (dir.), Pour une histoire du risque: Québec, France, Belgique, Montréal/Rennes, Presses de l'Université du Québec/Presses de l'Université de Rennes, 2012, p. 203-224.

4. Sur cette question, voir Martin PetiTClerC, "À propos de "ceux qui sont en dehors de la société". L'indigent et l'assistance publique au Québec dans la première moitié du XX $\mathrm{XX}^{\mathrm{e}}$ siècle", Revue d'histoire de l'Amérique française, vol. 65, $\mathrm{n}^{\text {os }} 2-3,2011$, p. 225-254.

5. Voir Martin PETITCLERC, «L'État providence, la société du risque et les fondements de la communauté politique", Martin PeTITCLERC et David NigeT (dir.), Pour une histoire du risque..., op. cit. et Martin PETITCLERC, "La construction du problème social de la maladie dans le Québec des années 1930 ", Marcelo OTERO et Shirley ROY (dir.), Qu'est-ce qu'un problème social?, Montréal, Presses universitaires du Québec, 2012, p. 229-248.

6. On sait peu de choses du père jésuite Léon Lebel (1883-1966). Après avoir grandi dans une famille rurale de 10 enfants à Cacouna et avoir étudié au Collège Sainte-Marie, il mène une carrière apostolique " consacrée successivement à trois grandes causes, l'enseignement, l'aumônerie de l'association professionnelle des cultivateurs et finalement la promotion des secours spirituels aux agonisants». Souffrant d'importants troubles de la vue, il devient progressivement aveugle, ce qui ralentit considérablement ses activités au sein de l'UCC, dont il est l'aumônier de 1929 à 1934, puis de 1937 à 1952. Voir Réal LEBEL, S.J., "L'apostolat joyeux du Père Léon Lebel (1883-1966)", Lettres du Bas-Canada, 1966, p. 216-229.

7. Voir notamment Léon LEBEL, Les allocations familiales: solution du problème des familles nombreuses, Montréal, École sociale populaire, $\mathrm{n}^{\text {os }} 159-160,1927$; Léon LEBEL, Pourquoi les allocations familiales? Raisons et objections, Montréal, Arbour et Dumont, 1928; Léon LEBEL, The Problem of the Large Family 
l'économie, du risque et de l'assurance sociale ${ }^{8}$. Considérant l'importance de la représentation de la famille dans la définition de l'ordre moral et social notamment pour un jésuite qui devait s'inscrire dans le cadre doctrinal des encycliques papales -, l'apparition d'un tel discours profane appliqué au problème "sacré » de la reproduction familiale est indéniablement un événement historique digne d'intérêt. Bien sûr, la campagne politique de Lebel pour des allocations familiales à la fin des années 1920 ne débouche sur aucune réforme à court terme. Toutefois, un système universel d'allocations familiales est mis en place en 1944 par l'État fédéral, bien que dans une forme différente de celle proposée par Lebel ${ }^{9}$. Cela dit, l'échec partiel de la campagne de Lebel n'enlève que peu de choses à sa signification historique : elle est un exemple éloquent de l'importance stratégique du savoir assurantiel et du discours sur le risque lors de la crise du capitalisme libéral au début du $\mathrm{XX}^{\mathrm{e}}$ siècle. Après avoir fait un survol de l'historiographie sur les allocations familiales et présenté en détail le projet de Lebel, nous réfléchirons sur les enjeux posés par ce dernier en regard de la genèse de l'État-providence au Québec.

\section{HISTORIOGRAPHIE}

Les allocations familiales, la première politique sociale universaliste au Canada, ont été abordées sous plusieurs angles. Certains historiens ont souligné que l'adoption de cette politique démontrerait «que la préoccupation du gouvernement pour le bien-être des citoyens venait d'atteindre un niveau supérieur ${ }^{10}$ ». Cela dit, les études n’ont pas tardé à montrer que les allocations familiales répondaient à divers objectifs qui n'avaient pas uniquement le bien-être des citoyens pour principal objectif. Ainsi, les allocations familiales ont été étroitement liées à la pacification des rapports

$$
\div+\div
$$

in Canada. Its Solution: Family Allowances, Montréal, [s.é.], 1928. Outre ces publications, Lebel rédige plusieurs articles de synthèse dans les journaux et revues nationalistes et catholiques, dont le quotidien $L e$ Devoir.

8. Selon Jean-Philippe Warren, les publications de Lebel sur les allocations familiales dans les années 1920 témoignent d'ailleurs de la montée d'un savoir sociologique qui se distinguerait d'une vision strictement théologique de la société (Jean-Philippe WARREN, L'engagement sociologique: la tradition sociologique du Québec francophone, 1886-1955, Montréal, Boréal, 2003, p. 62-63).

9. Comme nous le verrons un peu plus loin, l'historiographie a généralement exagéré l'opposition de Lebel au programme fédéral d'allocations familiales. Insensible à la question de l'égalité des hommes et des femmes à l'intérieur de la famille, Lebel est sans doute méfiant à l'égard d'un programme qui verse l'allocation directement aux mères. Cela dit, cette méfiance ne l'empêche pas d'appuyer ouvertement le programme et même de le défendre face aux critiques conservatrices catholiques et nationalistes. Voir notamment Léon LEBEL, Les allocations familiales au Canada, Montréal, Institut social populaire, 1951. 10. Dennis Guest, Histoire de la sécurité sociale au Canada, Montréal, Boréal, 1993, p. 187. 
sociaux dans le capitalisme industriel. Alors qu'on assistait à la montée d'un mouvement ouvrier plus revendicateur et d'un parti socialiste fédéral au cours de la Deuxième Guerre mondiale, les allocations familiales ont aussi été une stratégie afin de favoriser la paix industrielle et le développement du capitalisme ${ }^{11}$. Parallèlement, certaines historiennes féministes, en insistant sur l'étroite imbrication de la famille patriarcale au capitalisme industriel, ont soutenu que les allocations familiales ont permis le passage d'un "patriarcat familial » à un "patriarcat social ${ }^{12}$ ». Pour d'autres, les allocations familiales visaient moins à répondre aux nouveaux besoins du capitalisme industriel qu'à la montée du mouvement féministe. Ainsi, les allocations familiales s'inscrivaient principalement dans le cadre du déploiement des multiples stratégies de lutte contre l'émancipation des femmes dans la sphère privée de la famille, comme dans la sphère publique ${ }^{13}$. Au final, les allocations familiales, qui ont eu un impact indéniable sur l'amélioration des conditions de vie, apparaissent aujourd'hui comme une politique ambiguë qui a répondu à plusieurs intérêts et qui a été mise en place par un État qui en structurait l'application selon sa dynamique propre ${ }^{14}$.

Ces différentes perspectives ont coloré l'interprétation des historiens à l'égard de la contribution de Lebel, le principal promoteur des allocations familiales avant les années 1940. D’ailleurs, l'image qui se dégage de l'historiographie est loin d'être cohérente. Dennis Guest a vu chez Lebel l'un des premiers partisans d'une politique généreuse qui a dû subir les attaques à courte vue de travailleurs sociaux conservateurs, hostiles à toute forme d'intervention de l'État ${ }^{15}$. Dans une étude centrée sur les débats idéologiques au Québec, Peter Southam a également insisté sur la contribution de Lebel, rare partisan d'une "conception institutionnelle du bien-être " caractéristique de l'État-providence, face à un fort courant nationaliste conservateur et corporatiste qui défendait de son côté une « conception résiduelle du bien-

$$
\div \div
$$

11. Brigitte KITCHEN, "The Introduction of Family Allowances in Canada ", Allan MosCOVICH et Jim AlBert (dir.), The "Benevolent" State. The Growth of Welfare in Canada, Toronto, Garamond Press, 1987, p. 222-241. Voir également Alvin FInKel, Social Policy and Practice in Canada: A History, Waterloo, Wilfrid Laurier University Press, 2006, p. 130-135.

12. Jane Ursel, Private Lives, Public Policy: 100 years of State Intervention in the Family, Toronto, Women's Press, 1992.

13. Nancy CHRISTIE, Engendering the State: Family, Work, and Welfare in Canada, Toronto, University of Toronto Press, 2000.

14. Voir Dominique MARSHALl, Aux origines sociales de l'État-providence: familles québécoises, obligation scolaire et allocations familiales, 1940-1955, Montréal, Presses de l'Université de Montréal, 1998 et, plus récemment, Raymond Benjamin BLACK, From Rights to Needs : A History of Family Allowances in Canada, 1929-1992, Vancouver, UBC Press, 2009.

15. Dennis GUEST, Histoire de la sécurité sociale au Canada..., opt. cit., p. 117-119. 
être $^{16}{ }$. Dominique Marshall a également souligné que la proposition de Lebel, qui était indéniablement nataliste, reflétait tout de même une conception "sensiblement différente" de l'orthodoxie catholique relative à la doctrine sociale de l'Église. C'est pourquoi, «moins craintif envers l'État que l'épiscopat, [Lebel a] plaidé énergiquement en faveur d'allocations obligatoires et uniformes versées par les pouvoirs publics ${ }^{17}$ ». De leur côté, Danielle Gauvreau, Diane Gervais et Peter Gossage ont souligné que Lebel a rompu avec certains aspects du discours nationaliste clérico-nataliste en abordant la famille nombreuse comme un problème social et non selon les termes d'un idéal moral ${ }^{18}$. Chantale Quesney a défendu une position similaire en affirmant qu'une conception thomiste du politique, courante chez les jésuites, aurait permis à Lebel de formuler «une politique étatique d'aide à la famille qui se [voulait] globalisante, contredisant, en cela, une vision libérale du rôle économique de l'État ${ }^{19}$ ".

Certains historiens ont été nettement plus sévères à l'égard de Lebel et ont insisté sur la dimension conservatrice et patriarcale de sa contribution au débat sur les allocations familiales. Il faut dire ici que Lebel, après avoir été l'ardent défenseur des allocations familiales pour la classe ouvrière, devenait en 1929 aumônier général de l'UCC, organisation qui allait devenir un puissant syndicat rural corporatiste ${ }^{20}$. En 1940, quelques années après la reconnaissance du droit de vote des femmes, le père Lebel fondait l'Union catholique des fermières, une organisation qui visait à concurrencer les cercles des fermières qu'il considérait comme trop indépendants de l'Église ${ }^{21}$. Cet épisode témoigne indéniablement de l'antiféminisme de la pensée de Lebel. Andrée Lévesque a ainsi replacé la campagne de Lebel pour les allocations familiales dans le mouvement antiféministe plus large qui se radicalise dans les années $1920^{22}$. Denyse Baillargeon a, de son côté, souligné que le

$$
\div+\div
$$

16. Peter SOUTHAM, "Modernisation", "Question nationale " et influences exogènes: le discours élitaire sur les politiques sociales au Québec, 1930-1960, thèse de doctorat (histoire), Université de Montréal, 1987, p. 224.

17. Dominique MARSHALL, Aux origines sociales de l'État-providence..., op. cit., p. 41.

18. Danielle Gauvreau, Diane Gervais et Peter Gossage, La fécondité des Québécoises, 1870-1970: d'une exception à l'autre, Montréal, Boréal, 2007, p. 81-83.

19. Chantale QUESNAY, "Quand la maison signifiait quelque chose": la famille selon l'École sociale populaire, 1918-1939", Histoire Sociale, vol. 35, n 70, 2002, p. 479.

20. Jean-Pierre KESTEMEN et al., Histoire du syndicalisme agricole au Québec: UCC-UPA, 1924-2004, Montréal, Boréal, 2004.

21. Yolande COHEN, Femmes de parole : l'histoire des Cercles de fermières du Québec, 1915-1990, Montréal, Le Jour, 1990, p. 52 et suivantes.

22. Andrée LÉVESQUE, La norme et les déviantes: des femmes au Québec pendant l'entre-deux-guerres, Montréal, Éditions du Remue-ménage, 1989. 
projet d'allocations familiales [que Léon Lebel] défendait ne cherchait donc pas à faire reconnaître le travail maternel [gratuit], mais bien à valoriser la contribution sociale des pères prolifiques en leur permettant d'accéder à un niveau de revenu se rapprochant d'un «salaire familial ». [...] Le versement au père garantissant pour sa part le maintien de l'ordre patriarcal dans la famille ${ }^{23}$.

De son côté, Denyse Côté a souligné la critique de Lebel à l'égard de la politique libérale des allocations familiales de 1945 qui, selon lui, allait à l'encontre du droit des pères ${ }^{24}$. Enfin, c'est sans doute Nancy Christie qui a été la plus critique des propositions de Lebel. Pour elle, ce dernier était un corporatiste catholique de droite, antiféministe et profondément hostile à l'intervention de l'État:

Lebel's campaign for family allowances was studiously antifeminist in its implications; by his conception, both the family and the workplace were rightly dominated by the male breadwinner. Not only were family allowances intended as a "sur-wage" for the male worker, but they were also deemed to be financial recognition of the fact that the children belonged to the father. [...] By elevating male rights within the workplace and within the domestic sphere, Lebel was transgressing the principal articles of maternal feminism and at the same time rejecting the canons of progressive reforms ${ }^{25}$.

Les réflexions de Lebel sur les allocations familiales ont donc suscité plusieurs analyses, bien que l'image qui se dégage de l'historiographie soit au final plutôt confuse. Si celle-ci peut paraître "progressiste" ou "conservatrice " selon les chercheurs, c'est parce que ces derniers, qu'ils aient eu une vision positive ou négative de sa contribution, n'ont pas, jusqu'à ce jour, sérieusement pris en compte le fait que Lebel accordait une très grande importance au discours sur le risque pour justifier et définir les modalités d'application de sa politique. Et ce discours, qui s'appuyait sur un monde de justice inspiré des «lois» de l'économie politique, n'était évidemment pas neutre. Ainsi, bien qu'il prenne les allures de la science et de la technique, le discours sur le risque ne peut pas être considéré comme le simple véhicule d'une morale patriarcale inchangée ou d'une idée de la justice sociale à réaliser. En portant

$$
\div+\div
$$

23. Denyse BAillargeON, "Maternalisme et État-providence : le cas du Québec», Sextant, vol. 20, 2003, p. 113-147.

24. Denyse CÔTÉ, "Le versement des allocations familiales aux mères québécoises", Anita CARON et Lorraine ARChambault (dir.), Thérèse Casgrain. Une femme tenace et engagée, Montréal, Presses de l'Université du Québec, 1993, p. 226-227.

25. Nancy CHRISTIE, Engendering the State..., op. cit., p. 184-187. 
une plus grande attention aux fondements "assurantiels » du projet d'allocations familiales de Léon Lebel, on peut ainsi mieux comprendre tout à la fois les potentialités et les contraintes de ce discours pour la réforme sociale dans le contexte du capitalisme de l'entre-deux-guerres au Québec et au Canada.

\section{LEBEL ET LE MOUVEMENT DE RÉFORMES}

À la suite des activités de la modeste Société d'économie sociale de Montréal, c'est au sein d'un petit groupe de clercs, réunis sous le parapluie de l'ESP, qu'est porté le mouvement du catholicisme social avant la crise économique des années 1930. Au début des années 1920, l'École est appuyée par les Semaines sociales qui diffusent la "sociologie doctrinale " dans l'enseignement supérieur. Préoccupée par l'application des principes formulés dans l'encyclique aux conditions de la société canadienne-française, l'ESP s'engage alors dans la fondation d'associations de toutes sortes, à commencer par des caisses populaires, des coopératives, des associations de réformes et, enfin, des syndicats d'ouvriers et d'agriculteurs ${ }^{26}$. En 1921, la fondation de la Confédération des travailleurs catholiques du Canada vise ainsi à appliquer les principes de la doctrine sociale de l'Église aux relations de travail, après que de nombreux travailleurs canadiens-français se sont joints aux syndicats «internationaux» d'origine américaine et neutres sur le plan confessionnel. Quelques années plus tard, en 1924, l'UCC poursuit le même objectif en regroupant les agriculteurs canadiens-français. À cela s'ajoutent d'autres associations qui ne sont pas le produit direct du catholicisme social, mais qui en subissent indéniablement l'influence au cours des années 1920, comme la Fédération nationale Saint-Jean-Baptiste, la principale organisation féminine canadienne-française. À la fin des années 1920, que ce soit en collaboration ou en concurrence avec de nombreux autres regroupements laïques ou confessionnels, ces groupes sont à l'origine d'une importante campagne pour des réformes sociales au Québec.

Parmi la foule de mesures envisagées, les allocations familiales sont certainement l'une des réformes les plus discutées dans la seconde moitié des années 1920. Au moment de la campagne de Lebel, plusieurs pays européens, de même que certains pays du Commonwealth comme l'Australie et la Nouvelle-Zélande, ont déjà pris des initiatives variées en ce sens. En

$$
4+4
$$

26. Jean-Philippe WARREN, L'engagement sociologique: la tradition sociologique du Québec francophone, 1886-1955, Montréal, Boréal, 2003 p. 59-63. 
Europe continentale, les premières allocations familiales ont été adoptées volontairement par certains patrons de grande entreprise dès la fin $\mathrm{du}$ XIX ${ }^{e}$ siècle afin de lutter contre l'augmentation des salaires et la montée des organisations syndicales. Après les pertes humaines associées à la Première Guerre mondiale, un solide mouvement nataliste, voire nativiste, milite, en France et en Belgique notamment, pour une systématisation de ces expériences patronales, ce qui débouche à partir du début des années 1930 à des politiques natalistes d'allocations familiales obligatoires ${ }^{27}$.

Les racines idéologiques du mouvement des allocations familiales sont très différentes au Royaume-Uni et dans le Commonwealth. En Angleterre, la féministe Eleanor Rathbone revendique les allocations familiales depuis 1924, non pas comme une politique nataliste, mais bien comme un salaire pour le travail maternel "gratuit» des femmes. Comme le rappelle Susan Pederson, l'intérêt pour les allocations familiales provient ici de la gauche socialiste et féministe qui est préoccupée par la pauvreté de la famille ouvrière nombreuse et qui voit les allocations comme une façon de soutenir la demande interne, et donc l'emploi ${ }^{28}$. Toutefois, la campagne de Rathbone ne réussit pas à convaincre le mouvement ouvrier et le parti travailliste de la nécessité d'une telle politique, principalement parce que ces derniers craignent que des allocations familiales ne minent les revendications syndicales pour l'obtention d'un "salaire familial» (un salaire suffisant pour que le chef de famille puisse subvenir aux besoins de la mère et de leurs enfants). Au tournant des années 1930, le mouvement des allocations familiales en Angleterre est bloqué, principalement à cause des résistances patriarcales à l'intérieur du mouvement ouvrier et socialiste ${ }^{29}$.

Lebel est bien conscient de ces différences lorsqu'il commence à se prononcer pour des allocations familiales pancanadiennes dans une brochure de l'ESP intitulée Les allocations familiales. Solution du problème de la famille nombreuse. En plus d'en publier une version anglaise légèrement modifiée en $1928^{30}$, Lebel diffuse principalement ses idées au sein des milieux

$$
\div \div
$$

27. En France, le mouvement nataliste, d'inspiration nettement conservatrice, réussit même à interdire la "propagande néo-malthusienne " en 1920, ce qui vise explicitement le mouvement féministe qui se méfie de l'instrumentalisation de la maternité dans une politique nataliste, voire nativiste. Sur cette question, voir notamment Anna COVA, Maternité et droits des femmes en France, XIXe et XXe siècles, Paris, Anthropos, 1997 et Féminismes et néo-malthusianismes sous la III République: la liberté de la maternité, Paris, Harmattan, 2011.

28. Susan PEDERSON, Family, Dependence, and the Origins of the Welfare State: Britain and France, 19141945, Cambridge, Cambridge University Press, 1995, p. 178-179.

29. Ibid., p. 219 et suivantes.

30. Léon LEBEL, The Problem of the Large Family..., op. cit. 
catholiques, nationalistes et réformistes. En mai 1928, Lebel rencontre le premier ministre libéral Mackenzie King pour attirer son attention sur la pertinence d'allocations familiales. En février 1929, le député libéral fédéral J. E. Letellier réussit, avec l'appui du député ouvrier James $S$. Woodsworth, à convaincre la Chambre des communes que la question des allocations familiales soit abordée dans les travaux parlementaires du Select Standing Committee on Industrial and International Relations. De l'avis de tous, c'est Lebel qui apporte le principal témoignage pendant les trois mois que durent les travaux de ce comité parlementaire ${ }^{31}$. Encore en 1931, lorsque la Commission d'enquête sur les assurances sociales du Québec décide finalement d'étudier les allocations familiales, Lebel est convoqué pour y présenter son projet. Les commissaires affirment alors que,

bien que nos sociologues canadiens se soient intéressés grandement à cette forme d'aide à la famille nombreuse, il n'est pas téméraire de dire que, jusqu'à la campagne méthodique, active, tenace, menée en faveur des allocations familiales par le R. P. Léon Lebel, S. J., la question était restée dans le domaine d'une simple discussion spéculative, n'ayant aucunement pour objet d'introduire chez nous l'application qu'on en avait faite en Europe ${ }^{32}$.

\section{UN PROBLÈME D'ÉCONOMIE POLITIQUE}

En ouverture de son importante brochure de 1927 sur les allocations familiales, Lebel insiste sur le fait suivant:

Nous voudrions, dans ces quelques pages, montrer à tous ceux qui s'intéressent aux questions sociales, qu'il existe une question des familles nombreuses, que leur venir en aide est un devoir, non pas de charité, mais de véritable justice sociale, et qu'entre les maux dont souffre notre société, celui qui accable les familles nombreuses est un de ceux auxquels il faut en premier lieu porter remède. [...] En le résolvant, le pays, non seulement s'acquittera d'une dette de justice envers ses meilleurs serviteurs, mais il pourvoira à ses propres intérêts et s'assurera le meilleur facteur de prospérité qu'il saurait trouver ${ }^{33}$.

$$
\div+
$$

31. Raymond Benjamin BLACK, From Rights to Needs: A History of Family Allowances in Canada, 19291992, Vancouver, UBC Press, 2009, p. 24-25.

32. COMMISSION DES ASSURANCES SOCIALES DE QUÉBEC, Troisième et Quatrième rapports. Les allocations familiales, $2^{\mathrm{e}}$ édition, Québec, ministère du Travail, 1933, p. 90 et suivantes. Lebel est conscient des problèmes constitutionnels que poserait un programme fédéral d'allocations familiales. Il suggère de prendre le modèle des récentes pensions aux vieillards de 1927, c'est-à-dire un programme fédéral, à frais partagés, conditionnel à la participation des provinces. Ce programme est toutefois très contesté au Québec et le gouvernement provincial n'y participera qu'à partir de 1936 (Léon LEBEL, The Problem of the Large Family..., op. cit., p. 47).

33. Léon LEBEL, Les allocations familiales..., op. cit., p. 1-2. 
De cette dernière phrase, on a surtout retenu que Lebel considère les pères de familles nombreuses comme les «meilleurs serviteurs » du pays. Nous insisterons plutôt ici sur la "dette de justice", les "propres intérêts" du pays et le "meilleur facteur de prospérité » qui font référence au savoir profane de l'économie politique qui s'était développé en Occident depuis le milieu du XVIII ${ }^{\mathrm{e}}$ siècle et dont Adam Smith était l'un des plus illustres représentants. D'ailleurs, bien qu'elle constitue un passage obligé pour un jésuite de l'ESP, la doctrine sociale de l'Église ne joue finalement ici qu'un rôle secondaire. De toute façon, son projet d'allocations familiales ne s'adresse pas qu'aux seuls catholiques, mais bien à l'ensemble de la population canadienne. En fait, Lebel tente de réinterpréter les enseignements doctrinaux des encycliques à la lumière des principes fondamentaux de la «raison économique » sur lesquels doit s’appuyer la « véritable justice sociale». Lorsqu'il pose la question « Pourquoi les allocations familiales?», Lebel rappelle ainsi l'importance

d'en exposer la raison économique, en montrant la situation anormale faite à la famille nombreuse par la civilisation moderne. En effet, la question des allocations familiales n'est pas une question isolée qui peut se traiter à part. Elle se rattache intimement à celle du salaire et celle-ci fait elle-même partie de la question plus vaste encore du régime économique ${ }^{34}$.

Selon Lebel, ce problème "plus vaste " découle des conditions de vie produites par la «double révolution sociale et économique de la fin du XVIII siècle». Il dénonce alors les conditions sociales « anormales» de son époque, "où le travail humain est considéré comme une marchandise quelconque et d'après laquelle le travailleur doit être abandonné, faible et isolé, en face du patron et du capitaliste tout-puissants ${ }^{35}$ ». Selon Lebel, le salariat s'est imposé par le processus historique « d'une expropriation [de la propriété familiale qui] s'est faite insensiblement au cours des années; [qui a été] le résultat d'une lente évolution dans le régime économique [et] qui a amené une rupture d'équilibre entre les citoyens ${ }^{36}{ }$. Cette rupture d'équilibre aurait modifié la nature même du contrat social patriarcal, qui est selon Lebel le produit de l'entente entre les pères pourvoyeurs qui désirent se donner collectivement les moyens économiques pour assurer leur propre subsistance ainsi que celle de leurs personnes à charge. Étant donné que cette expropriation aurait

$$
4 \div 4
$$

34. Ibid., p. 2-6.

35. Ibid., p. 6.

36. Ibid., p. 30. 
modifié les termes du contrat social patriarcal, l'État aurait donc «le devoir de rétablir l'égalité détruite par le changement des conditions de vie; il [doit] faire en sorte que les biens nécessaires à l'existence soient répartis entre citoyens suivant la proportion qu'exigent le mérite et la justice». Pour Lebel, l'État doit intervenir dans l'économie afin que celle-ci soit «apte à procurer dans la plus grande mesure possible le bien terrestre de tous les sociétaires", entendus ici comme les membres d'une communauté politique laïque d'hommes pourvoyeurs ${ }^{37}$.

Cette intervention de l'État pose toutefois un problème délicat pour Lebel, qui doit, malgré tout, s'inscrire dans les cadres généraux de la doctrine sociale de l'Église. Or celle-ci privilégie, comme l'essentiel du mouvement ouvrier d'ailleurs, le respect du principe d'un salaire proportionnel aux charges familiales, ce qu'on appelle le "salaire familial ». Toutefois, selon Lebel, une telle conception du salaire familial, qui rendrait les allocations familiales inutiles, est contraire aux lois les plus fondamentales de l'économie, et notamment à celle qui stipule qu’à "travail égal, salaire égal ». Selon Lebel, les sociologues catholiques auraient erré en interprétant d'une façon trop littérale la doctrine sociale de l'Église. Selon lui, le salaire familial doit plutôt être envisagé par rapport au coût global de reproduction de la maind'œuvre, et donc suffire à la reproduction de la «famille moyenne». Entendue ainsi, la notion de salaire familial signifie simplement qu'un salaire devrait normalement être suffisant pour « au moins maintenir le chiffre de la population; c'est-à-dire [maintenir] la famille composée de cinq personnes : le père, la mère et trois enfants ${ }^{38}$ ".

En fait, la notion de salaire familial est moins une voie de solution pour la réforme sociale qu'une notion permettant de mieux comprendre le problème d'économie politique que pose la famille nombreuse dans les conditions sociales du début du XX $\mathrm{X}^{\mathrm{e}}$ siècle. D'une part, Lebel n’a aucune difficulté à démontrer, en s'appuyant sur les statistiques du ministère du Travail, que le salaire moyen des ouvriers (959 \$ par année) ne permet pas aux pères de subvenir au coût de la reproduction de la «famille moyenne», c'est-à-dire de payer les frais de nourriture, de chauffage et de loyer d'un ménage de cinq personnes (évalués à 1113 \$ en 1926). D'autre part, et c'est là l'important pour Lebel, même dans les conditions sociales idéales (et improbables) du salaire familial, les pères de famille nombreuse seraient tout de même

$$
4+4
$$

37. Ibid., p. 5-7, 12, 30.

38. Léon LEBEL, Pourquoi les allocations familiales..., op. cit., p. 8-10. 
désavantagés, puisque les lois de l'économie interdisent que le salaire puisse être augmenté pour couvrir les charges familiales supplémentaires. À l'inverse, le travailleur célibataire bénéficierait d'une relative aisance puisqu'il recevrait le salaire proportionnel au ménage moyen, mais sans assumer des charges familiales équivalentes. Puisque le niveau des salaires ne peut être ajusté en fonction des charges familiales de l'un ou de l'autre sans contrevenir aux lois du marché du travail, le problème qui frappe « le cas des familles nombreuses [doit] être résolu autrement que par les salaires ${ }^{39}$ ". Dit autrement, ce n'est pas le travail salarié, mais bien le travail familial non rémunéré de reproduction qui doit donner droit aux allocations ${ }^{40}$. En cela, même s'il ne reconnaît pas l'autonomie de la mère dans la famille patriarcale, Lebel contribue néanmoins à un élargissement considérable du concept de travail productif, habituellement limité au travail rémunéré.

C'est pourquoi Lebel définit les allocations familiales comme un "supplément proportionnel au nombre d'enfants dont [les soutiens de famille] ont actuellement la charge ${ }^{41}$ ». Une telle définition des allocations familiales découle de sa conception particulière du problème de la famille nombreuse, soit ce qu'il considère comme l'injuste vulnérabilité des chefs de famille nombreuse face au risque de charges familiales trop lourdes. Comme il le souligne au tout début de sa brochure anglaise: "Among the causes which have contributed to bring about this unfortunate state of affairs in social economies, we would place in the first rank the unequal distribution of family burdens among adults ${ }^{42}$ ". C'est contre ce risque économique de charges sociales trop élevées qu'il faut selon lui protéger les pères qui ont de nombreuses personnes à charge.

\section{LE RISQUE DE LA FAMILLE NOMBREUSE}

Interpréter les charges de famille en termes de risque pour le capital familial implique toutefois que les parents n'aient aucune responsabilité personnelle quant au nombre de naissances que produirait leur ménage. En effet, le risque fait référence à un événement aléatoire, un «accident " indépendant de la volonté personnelle. Pour Lebel, qui s'oppose évidemment en tant que membre du clergé à toute planification et limitation des naissances, la taille

$$
\div+\div
$$

39. Ibid., p. 12-13. Pour une discussion plus approfondie du niveau de vie au Canada, voir Léon LEBEL, The Problem of Large Family..., op. cit., p. 23 et suivantes.

40. "Thus when the breadwinner is ill and so receives no pay he still has a right to the allowance which certainly cannot be called a sur-wage" (Léon LEBEL, The Problem of Large Family..., op. cit., p. 17).

41. Léon LEBEL, Les allocations familiales..., op. cit., p. 12-13.

42. Léon LEBEL, The Problem of Large Family..., op. cit., p. 3. 
des ménages ne relève pas de la volonté des parents, mais bien de celle de la providence ${ }^{43}$. Après tout, la naissance d'un enfant dans tel ménage plutôt que dans tel autre est un événement qui, bien que relevant selon Lebel de la volonté providentielle, n'en est pas moins rigoureusement aléatoire sur le plan statistique. C'est donc dire que les charges familiales associées à la naissance d'un enfant peuvent être présentées comme un risque, au même titre que ceux associés aux charges sociales aléatoires que sont la maladie, la vieillesse, le chômage, etc. Lebel cite ainsi l'économiste jésuite belge Valère Fallon:

L'assimilation se justifie, puisque les charges de famille sont, elles aussi, des charges normales, c'est-à-dire légitimes et même hautement souhaitables pour le bien de la société: il doit donc y être pourvu de toute nécessité; elles sont certaines pour l'ensemble d'une population, et leur taux est même plus exactement connu que celui des autres aléas sociaux; elles sont très inégales, surtout dans l'état social actuel; elles sont très onéreuses pour ceux auxquels elles adviennent; enfin, elles sont aléatoires, dépendant de diverses circonstances indépendantes de la volonté... ${ }^{44}$

Et comme toute charge sociale, les coûts d'une famille nombreuse peuvent être estimés. Cela nécessite d'abord d'évaluer ce que représente la valeur d'une personne pour la société. Parmi tous les facteurs de production (la nature, le capital et l'homme), insiste-t-il, l'homme est certainement la plus importante source de plus-value ${ }^{45}$. Lebel rappelle ainsi que "le citoyen, produisant tout le cours de sa vie une certaine quantité de richesses qui accroît d'autant la valeur du pays, est un capital à intérêt; et [nous pouvons calculer] ce qu'il vaut à la société humaine, estimé en espèces ${ }^{46}$ ». Ce calcul est rendu possible par la production des statistiques par les compagnies d'assurances. Ainsi, Lebel affirme qu'un « homme de trente ans, qui gagne \$50,00 par semaine, a une valeur économique de $\$ 31000$, et que déjà à sa naissance un enfant représente pour le pays une valeur de $\$ 9,333 »$. Conscient que cette valeur tirée de statistiques américaines est probablement trop élevée pour correspondre aux conditions sociales du Canada, Lebel l'abaisse à $5000 \$$ pour rendre compte du plus faible niveau de vie. Il en résulte tout de même que la contribution du père de famille à la société, pour un ménage comp-

$$
+4 \div
$$

43. Léon LEBEL, Les allocations familiales..., op. cit., p. 26-27.

44. Valère Fallon, cité dans Léon LEBEL, Les allocations familiales..., op. cit., p. 52-53.

45. Léon LEBEL, The Problem of Large Family..., op. cit., p. 11.

46. Léon LEBEL, Les allocations familiales..., op. cit., p. 26-27. 
tant 10 enfants, est selon lui l'équivalent d'un investissement en capital de $50000 \$ 47$.

En soi, un tel investissement dans la société ne place pas nécessairement les familles dans une situation de vulnérabilité. Par exemple, un enfant dans les familles paysannes représente un "actif " qui contribue parallèlement à faire fructifier le capital du ménage par son travail dans l'entreprise familiale. La famille paysanne bénéficie directement de l'investissement qu'elle fait dans la société, par l'intermédiaire de la contribution de l'enfant à la constitution du capital familial. Mais les conditions sociales du capitalisme ont fait passer l'enfant de la famille salariée de la colonne des actifs vers celle des passifs dans les états financiers familiaux. En effet, étant donné les exigences du travail industriel, les normes plus élevées d'éducation, les lois pour interdire le travail juvénile (autant de réformes que Lebel considère comme justes et nécessaires), l'enfant ne peut plus contribuer au capital d'une famille salariée. Pour celle-ci, l'enfant ne représente plus un «actif» économique productif, mais bien un "passif " qu'il faut nourrir, héberger, soigner, éduquer, etc.

Il s'agit donc des charges familiales aléatoires évoquées plus haut qui, grâce encore aux statistiques des compagnies d'assurance, peuvent être évaluées. Reprenant l'exemple d'une famille de dix enfants, ces statistiques permettent d'évaluer ces charges à 7200 \$ par enfant aux États-Unis, valeur que Lebel réduit à $4000 \$$ afin de rendre compte du plus bas niveau de vie au Canada. Lebel constate alors une injustice flagrante: «il s'ensuit cette anomalie que, pour faire à la société un cadeau de $\$ 50000$, un père de dix enfants est obligé de dépenser $\$ 40000^{48}$ ». Puisque les pères de famille nombreuse (4 enfants et plus, soit un de plus que la famille moyenne), qui ne représentent que $20 \%$ de l'ensemble des ménages, entretiennent un peu moins de la moitié de tous les enfants, l'investissement est un bien mauvais risque: "Ainsi les services rendus à la société par le père de famille nombreuse se retournent contre lui et l'acculent à la misère ${ }^{49}$ ». Sur le plan de la stricte rationalité économique, la famille salariée devrait donc éviter d'engendrer des enfants...

$$
4+4
$$

47. Léon LEBEL, Les allocations familiales..., op. cit., p. 17. L'année suivante, il affirme que les statisticiens de la Dominion Life Assurance Co. ont démontré que la valeur d'un homme de 35 ans, gagnant 50 \$ par semaine, est de 36982 \$ (Léon LeBel, The Problem of Large Family..., op. cit., p. 12).

48. Léon LEBEL, Les allocations familiales..., op. cit., p. 17 et 21.

49. Ibid. 
Rejetant le malthusianisme, qui postule que l'accroissement de la population au-delà des ressources limitées du territoire est la principale source de la misère, Lebel considère, tout comme Adam Smith ${ }^{50}$, que le nombre est un facteur constant de prospérité. Selon Lebel, les statistiques démontrent que la croissance de la population, essentielle au dynamisme de la société, exige des familles moyennes au moins trois enfants. Mais puisqu'il s'agit d'une moyenne, et qu'il faut compter les célibataires et les familles avec peu d'enfants, c'est donc dire que les familles nombreuses (de plus de trois enfants) sont une nécessité économique, statistique et providentielle. Sans l'apport de celles-ci, la société manquerait tout simplement de travailleurs, de consommateurs, de contribuables, etc., qui sont la principale source d'enrichissement de la société. Or, puisqu'on peut démontrer que les pères pourvoyeurs assument de lourdes charges sans contrepartie, Lebel en conclut logiquement que leur investissement dans la société profite à d'autres, que ce soit aux industriels, aux commerçants, aux pouvoirs publics, etc. Ces derniers, qui profitent du travail de reproduction non rémunéré, ont donc une "dette » économique à l'égard des familles nombreuses. Il faut donc réfléchir, selon Lebel, à un mécanisme permettant le remboursement de cette dette contractée par les personnes, les groupes et organisations qui bénéficient directement de cet investissement. Cela peut-il être la charité, la forme traditionnellement privilégiée de redistribution des richesses dans l'imaginaire chrétien ? Non, croit Lebel, dans la mesure où nous sommes dans l'univers d'une transaction strictement économique, et donc dans le monde de justice profane de l'économie politique. Ainsi, il rappelle que même les patrons catholiques français, bien qu'ils se réclament de la charité catholique, ont volontairement implanté les allocations familiales pour une seule raison: ils y ont trouvé leur compte ${ }^{51}$. En fait, la rationalité de la charité, qui relève d'un monde de justice tout à fait différent de l'économie politique, risque plutôt de travestir ce qui relève d'abord et avant tout de la logique d'un droit contractuel qui lie ceux qui ont des parts dans la société, soit les sociétaires pourvoyeurs. Bien sûr, Lebel ne rejette pas la charité qui convient aux «cas anormaux " qui prennent la forme de l'indigence, soit un état de misère et de

$$
4+4
$$

50. Dans la version anglaise de sa brochure, il présente ainsi le problème qui l'intéresse: "One has but to turn to the pages of the Bible to ascertain that the birth of a child is ever represented as a legacy bequeathed by God to those who serve Him; and modern economists agree, with Adam Smith, that a high birth-rate is the most convincing sign of the prosperity of a country" (Léon LEBEL, The Problem of Large Family..., op. cit., p. 3).

51. Léon LEBEL, Les allocations familiales..., op. cit., p. 39. 
dépendance extrême ${ }^{52}$. Mais Lebel, qui s'appuie sur la statistique sociale pour démontrer la contribution nécessaire de la famille nombreuse au régime démographique, considère que cette dernière ne relève pas de l'anormalité, mais plutôt de la normalité. L'allocation familiale pour la famille nombreuse doit donc reposer sur un autre mode de redistribution de la richesse : l'assurance.

\section{L'ASSURANCE FAMILIALE}

Comme l'a souligné François Ewald, l'assurance est fille du capital ${ }^{53}$. Une fois les charges familiales considérées comme un investissement du capital familial dans la société, Lebel peut ensuite envisager la mutualisation des risques de cet investissement social sous la forme de l'assurance. Tout comme Fallon, Lebel considère alors qu'on peut assimiler «les charges de famille aux autres charges sociales aléatoires auxquelles il est pourvu par les diverses assurances : vieillesse, maladie, invalidité, veuvage, accident, chômage ${ }^{54} »$. Les modalités administratives de cette assurance familiale constituent, toutefois, «la partie la plus difficile» de son projet. Les problèmes sont nombreux : l'assurance devrait-elle être volontaire ou obligatoire? Qui devrait contribuer aux allocations? Quels seraient les coûts d'une telle politique? Devrait-on indemniser le père dès le premier enfant? Toutes les familles devraient-elles être couvertes? Qui devrait administrer les fonds? Toute une série de problèmes qui trouvent, selon Lebel, leur solution dans une conception de la solidarité que l'on peut dégager du fonctionnement même de l'assurance, qui apparaît de plus en plus comme le mode privilégié de gestion des inégalités matérielles dans le régime capitaliste.

Énoncé brièvement, il s'agit pour Lebel de répartir le risque que représentent les charges sociales de la famille nombreuse sur l'ensemble des parties de la société qui bénéficient de cet investissement. La question est alors de savoir si cette répartition doit se faire d'une façon volontaire ou obligatoire. D'abord, bien que l'histoire des allocations familiales montre que celles-ci ont d'abord été impulsées d'une façon volontaire par les grands patrons d'entreprises, Lebel considère néanmoins qu'il faudrait recourir à l'assurance publique obligatoire. Et cela, pour des raisons d'ordre économique : un patron qui paierait volontairement des allocations familiales

$$
4+4
$$

52. Selon la loi de l'assistance publique, l'indigence était définie comme l'incapacité physique (ou mentale) de subvenir à ses propres besoins et l'absence de tout soutien provenant de la famille élargie.

53. François EWALD, L'État providence, Paris, Grasset, 1986, p. 182.

54. Valère Fallon, cité dans Léon LEBEL, Les allocations familiales..., op. cit., p. 52-53. 
aurait de la difficulté à concurrencer un autre patron qui ne les paierait pas. De plus, les pères de famille nombreuse auraient avantage à aller dans les usines du premier, augmentant ainsi ses charges d'entreprise au point de l'acculer à la faillite, alors que le second n'aurait recours qu'à de jeunes travailleurs célibataires, ce qui lui permettrait ainsi d'exercer une pression à la baisse sur les salaires. Bref, et même lorsqu'on envisage des caisses de compensation étendues à l'ensemble d'un secteur de l'économie, ce type d'allocations familiales se retournerait invariablement contre les pères de famille nombreuse. La "raison économique» pointe donc vers « une loi rendant les allocations uniformes et obligatoires pour tous les salariés ${ }^{55}$.

Cela dit, même s'ils sont les premiers intéressés, les patrons ne sont pas les seuls à profiter matériellement de la famille nombreuse. La répartition des risques doit donc être élargie bien au-delà de ce groupe social. Comme le souligne Lebel, «le père de famille ne mérite pas seulement des employeurs [...] c'est la société tout entière qui est engagée envers lui. Elle doit donc contribuer à alimenter les caisses de compensation suivant un certain pourcentage que déterminera une étude approfondie de la ques$\operatorname{tion}^{56}$ ". Dans son projet, Lebel évalue que les dépenses annuelles d'allocations, qui représentent l'équivalent d'environ 1,5\% de la somme des salaires, seraient financées à partir des primes des trois niveaux de gouvernements (30\%), des employeurs (20\%) et de certaines catégories de citoyens, «dont les célibataires et les mariés sans enfant seraient les principaux contribuants " (50\%). Cette " taxe " à l'égard des célibataires et des parents sans enfant peut évidemment être interprétée comme une politique punitive de moralisation visant à imposer le modèle de la famille nombreuse. Sans nier que cette conception nataliste ait pu jouer, il faut toutefois noter que Lebel s'en remet exclusivement à la logique de l'assurance pour expliquer cette redistribution sociale des richesses des célibataires vers les familles «victimes» de l'accident providentiel de la famille nombreuse :

La taxe ainsi payée par le jeune homme pendant les années qui précèdent son entrée en ménage et la naissance d'un enfant, [sic] serait assimilable à une prime d'assurance dont il toucherait le bénéfice dès qu'il aurait des enfants. [...] Mais, dira-t-on, plusieurs d'entre ces célibataires n'auront peut-être jamais d'enfants ou ne recevront en allocations qu'une partie du montant qu'ils auront versé. Nous répondons que ce risque est dans la logique de l'assurance. Ne paie-t-on

$$
4 \div
$$

55. Léon LEBEL, Les allocations familiales..., op. cit., p. 39-41.

56. Ibid., p. 40. 
pas pendant des années des primes contre l'incendie, alors qu'on espère bien en être préservé et que de fait l'on n'en subit jamais ${ }^{57}$ ?

Lebel croit que la logique de la protection assurantielle mène directement à un système universel d'allocations familiales pour l'ensemble des ménages :

On ne saurait nier qu'un des principes qui ont donné naissance aux allocations conduise logiquement à les généraliser. Puisque celles-ci sont une rémunération des services rendus à la société et aux employeurs par le père de famille, il faut conclure que tout père de famille y a droit, puisque tous sont égaux dans la prestation de ce service $^{58}$.

Sur le plan des principes, Lebel est donc favorable à l'extension des allocations aux familles paysannes ${ }^{59}$, mais considère que les familles ouvrières, les plus vulnérables face au risque des charges familiales élevées, devraient d'abord être privilégiées ${ }^{60}$. La même logique de l'assurance laisse également entrevoir que des prestations puissent être versées à tous ceux qui sont aux prises avec des charges familiales, et ce à partir du premier enfant. En exagérant l'importance de la dimension nataliste dans le projet de Lebel, l'historiographie n'a pas vu que si celui-ci favorise les familles de plus de trois enfants, cela résulte au moins autant de la logique du risque et de l'assurance que d'une stricte adhésion à l'idéologie nataliste. Voici comment Lebel présente les choses :

il se peut qu'après avoir étudié le coût probable des allocations et les ressources dont on dispose, on en vienne à la conclusion qu'il serait trop onéreux de commencer à servir des allocations dès le premier enfant. On pourrait dans ce cas laisser provisoirement de côté le premier, ou même le deuxième enfant. Mais, comme il est avéré que le salaire général au Canada est insuffisant pour une famille de cinq, il faudrait commencer au moins dès le troisième. Cette mesure permettrait peut-être de donner pour les enfants suivants, une allocation plus substantielle ${ }^{61}$.

$$
\div+\div
$$

57. Ibid., p. 54-55. Voir également Léon LeBEL, The Problem of Large Family..., op. cit., p. 50-51.

58. Léon LEBEL, Les allocations familiales..., op. cit., p. 42.

59. Au sujet de la situation des familles paysannes, Lebel affirme qu'il «n'y a plus guère que les poètes à célébrer les charmes de la campagne ". L'enjeu est évidemment fondamental pour la question de l'exode rural, un problème politique récurrent depuis le milieu du XIX ${ }^{\mathrm{e}}$ siècle au Québec: "On ne pourra empêcher le campagnard, doué de sens commun, de se servir de sa logique: puisque les allocations sont données comme une rémunération des services que rendent au pays ceux qui lui fournissent des citoyens nouveaux, le père de famille agriculteur en conclura qu'il y a lui-même le premier droit. Si on les lui refuse à la campagne, il ira chercher pleine satisfaction de son droit dans les villes » (ibid., p. 42-44).

60. Ibid., p. 49.

61. Ibid., p. 51 . 
Enfin, la logique redistributive de l'assurance permet de se dégager des accusations de prôner un socialisme d'État ou un paternalisme d'État, toujours susceptibles de miner les projets de réforme au début du XX siècle. "Le problème le plus délicat à résoudre, affirme Lebel, sera celui de l'organisation à donner aux caisses d'allocations». Il privilégie "une vaste caisse nationale d'allocation comme l'unique solution pratique » qui permettrait éventuellement d'offrir des allocations familiales à « toutes les catégories de citoyens ». Quant à la gestion de la caisse, Lebel envisage qu'elle «soit dirigée par des représentants de tous les intéressés, qu'elle jouisse même, à la façon d'autres commissions déjà existantes chez nous, d'une certaine autonomie, qui la rende indépendante de la politique ${ }^{62} »$. En s'appuyant sur l'objectivation du risque des charges familiales et la logique redistributive de l'assurance, le projet de Lebel témoigne ainsi du potentiel de ce discours pour justifier des réformes sociales dans le cadre d'un monde de justice déduit des règles de l'économie politique.

Est-ce que l'assurance familiale est du socialisme ? C'est là une mauvaise question selon Lebel. En effet, «dès lors que l'on vit en société, il faut de toute nécessité qu'il y ait du socialisme, c'est-à-dire coopération des efforts de tous et entr'aide [sic] pour le bien commun. Les sociétés d'assurances et de secours mutuels, les coopératives, etc., sont du socialisme, mais du bon et qu'il faut encourager ${ }^{63}{ }^{\prime}$. Le mauvais socialisme, condamné justement par l'Église selon lui, est celui qui remet en question la propriété privée et qui encourage le relâchement des liens familiaux, entre époux et épouse, entre parents et enfants ${ }^{64}$. À cet égard, Lebel a bien compris que la socialisation des risques de la famille nombreuse par l'assurance n'implique aucunement une intervention moralisatrice de l'État dans les familles. Comme l'a souligné Ewald, les solidarités assurantielles «ne saisissent les individus que dans l'abstraction de leurs risques» contrairement aux solidarités organiques, comme la famille ou la paroisse, auxquelles «on appartient, dans la mesure où l'on respecte les devoirs, les hiérarchies, l'ordre qui sont les leurs ${ }^{65}$ ".

C'est bien en saisissant les pères de famille dans l'abstraction de leurs risques que Lebel peut affirmer que son projet d'allocations n'a " rien de commun avec l'étatisme ou le socialisme", en ce qu'il ne contraint ni la volonté des familles ni celle de la providence. En cela, illustrant en quoi le

$$
+4+
$$

62. Ibid., p. 51-53.

63. Léon LeBEL, Pourquoi les allocations familiales? Raisons et objections, Montréal, Arbour et Dumont, 1928 , p. 21.

64. Ibid., p. 21-22

65. François EWALD, Histoire de l'État providence, Paris, Grasset 1986, p. 177. 
projet de Lebel a pu apparaître à la fois "conservateur » et "progressiste » aux historiens, rien n'empêche que la socialisation des risques économiques de la famille nombreuse puisse se faire parallèlement à la refondation morale de la famille organique :

quant à la responsabilité morale, à la direction à donner à cette éducation, elles resteront la part exclusive des parents. L'intervention de l'État par les allocations n'implique nullement le droit de s'immiscer dans le gouvernement intérieur de la famille, qui est et doit rester le domaine sacré du père et de la mère. En leur facilitant les moyens de remplir leurs devoirs envers leurs enfants, l'État ne fait que remplir son rôle primordial qui est de suppléer à l'insuffisance des citoyens ${ }^{66}$.

\section{CONCLUSION}

Comme nous l'avons souligné, la campagne de Lebel pour des allocations familiales, malgré les efforts déployés, n’a pas connu de succès politique à court terme. En 1930, le décret mettant sur pied la Commission sur les assurances sociales de Québec mentionne vaguement «l'assistance aux familles nombreuses " plutôt que les allocations familiales promues par Lebel. Aussitôt le décret connu, Lebel fait circuler une pétition afin de demander à la commission d'accorder la primauté à l'étude des allocations familiales. Cette pétition, qui rappelle les thèmes abordés dans les brochures de Lebel, exige que les allocations familiales constituent «le centre, [...] le pivot ou l'axe de tout le système d'assurances sociales que l'on s'apprête à établir ${ }^{67}$ ». Si la pétition force les commissaires à y consacrer bien plus de temps que prévu, la suite est très décevante pour Lebel et ses partisans : le projet d'allocations familiales est le seul, de tous les projets d'assurances sociales envisagés (assurance chômage, assurance vieillesse, assurance maladie...), qui ne soit pas recommandé par les commissaires, même à titre expérimental.

Bien qu'ils reconnaissent que les allocations familiales sont une «solution au problème si important des familles nombreuses" en Europe, les commissaires craignent qu'elles ne mènent à une diminution des salaires, à une diminution des profits dans l'industrie, à une accélération de l'exode rural et à l'affaiblissement moral de la population. Les commissaires proposent plutôt une augmentation des salaires pour les ouvriers, une "politique inventive de colonisation» et des assurances sociales qui protégeraient non

$$
4 \div 4
$$

66. Léon LEBEL, Les allocations familiales..., op. cit., p. 56.

67. La pétition est reproduite en entier dans COMMISSION DES ASSURANCES SOCIALES DE QUÉBEC, Troisieme et Quatrième rapports.., op. cit., p. 92-94. Pour les recommandations, voir p. 107-109. 
pas le travail familial de reproduction comme le veut Lebel, mais bien le travail salarié des hommes pourvoyeurs en cas de chômage, de vieillesse ou de maladie. Pour les mères indigentes accidentellement privées du soutien d'un homme pourvoyeur, à la suite d'un décès ou d'une maladie mentale, et donc privées de la protection d'un éventuel système d'assurances sociales, elles seraient secourues par la charité publique, c'est-à-dire les pensions aux mères nécessiteuses ${ }^{68}$. Même s'il ne s'est pas réellement prononcé sur ces propositions, on peut penser que Lebel n'y était pas hostile. Cela dit, l'ensemble de ces mesures, selon Lebel, ne s'adresse pas à ce qu'il considère comme l'injustice la plus flagrante, soit celle de la misère de la famille ("normale») nombreuse, dont l'état de pauvreté découle de charges familiales trop lourdes engendrées par les conditions spécifiques du capitalisme industriel.

Quoi qu'il en soit, ce rejet du projet d'allocations familiales de Lebel illustre bien qu'il ne suffisait pas de reformuler les problèmes sociaux en termes d'accident pour légitimer politiquement une nouvelle proposition de réforme. Comme l'a bien souligné l'historiographie féministe, les propositions de réformes doivent également être légitimes du point de vue des normes identitaires de genre, qui sont rarement contestées à cette époque, même par les femmes réformistes ${ }^{69}$. Il faut rappeler qu'en élargissant le concept de travail rémunéré à celui du travail non rémunéré de reproduction, Lebel « découvre» ainsi le travail reproductif familial, même s'il n'admet pas que ce travail puisse légitimer, comme c'était le cas dans le projet de Rathbone par exemple, l'émancipation des mères à l'intérieur de la famille. Cette "découverte" permet ensuite d'envisager un droit à la protection sociale qui n'est pas directement arrimé au travail salarié du pourvoyeur, mais plutôt au travail non rémunéré de reproduction exécuté par la famille, entendue ici comme la cellule de base indivisible de la société. Dans sa pétition envoyée à la commission, Lebel explique ainsi pourquoi il considère que les allocations familiales doivent être prioritaires: «l'on ferait erreur en adoptant un système d'assurance individualiste [c'est-à-dire centré sur l'homme pourvoyeur et le travail salarié], sans songer d'abord et avant tout à la famille déjà trop méconnue et oubliée ${ }^{70}$ ». Pour rejeter le projet de Lebel, les commissaires

$$
\div+\div
$$

68. Sur cette question, voir Yolande COHEN, Femmes philanthropes: catholiques, protestantes et juives dans les organisations caritatives au Québec, 1880-1945, Montréal, Presses de l’Université de Montréal, 2010. 69. Linda GORDON, "Social Insurance and Public Assistance: The Influence of Gender in Welfare Thought in the United States, 1890-1935", The American Historical Review, vol. 97, n 1, 1992, p. 1954.

70. Voir CASQ, Troisième et Quatrième rapports. Les allocations familiales, $2^{\mathrm{e}}$ édition, Québec, ministère du Travail, p. 92-94. Bien sûr, Lebel n'a jamais remis en question la vision patriarcale de la famille dans 
s'appuient sur l'argumentation ultralibérale, malthusienne et patriarcale de la travailleuse sociale Charlotte Whitton. Cette dernière, qui a eu un impact bien plus important que Lebel sur les débats de la commission, craint que les allocations familiales interfèrent avec le pouvoir patriarcal de l'homme pourvoyeur à l'intérieur du foyer familial. Elle affirme ainsi

qu'abandonner ce principe [de l'obligation imposée au chef de famille de protéger ses enfants], laisser abroger cette obligation ou permettre que l'État l'assume ou en partage la responsabilité, c'est vouer à la destruction la base même sur laquelle repose notre conception de la vie de famille et de la protection de l'enfance ${ }^{71}$.

Paradoxalement, alors que l'historiographie féministe a insisté sur la nature patriarcale du projet de Lebel, c'est bien au nom des valeurs patriarcales que celui-ci est rejeté dans les années 1930.

Cela dit, si le projet assurantiel de Lebel ouvre effectivement des perspectives pour une mutualisation des risques des charges familiales, à condition d'accepter que la naissance soit, en l'absence de toute régulation des naissances, un "accident providentiel » qui ne relève pas de la volonté de la communauté conjugale, celui-ci a néanmoins pour conséquence de limiter étroitement le territoire de la solidarité. D'une part, comme nous l'avons souligné, son projet s'inscrit toujours dans une communauté politique où le chef de famille (masculin) est le seul sociétaire légitime, rejetant ainsi les prétentions justifiées de nombreuses femmes à être reconnues inconditionnellement comme des détentrices d'une part égale de la souveraineté politique. D'autre part, comme nous l'avons également rappelé, le discours sur le risque et l'assurance est un discours qui s'appuie sur le monde de justice de l'économie politique. D'où l'importance de montrer que la reproduction familiale est également un travail, dont la valeur peut être évaluée en espèce. Ce n'est donc pas un droit inconditionnel qui aurait relevé, par exemple, de l'appartenance de tous et de toutes à une même communauté politique, mais bien d'un droit conditionnel à l'investissement (en travail de production ou

$$
4+4
$$

le code civil. Dans une série de neuf articles publiés dans Le Devoir entre le 15 mars et le 16 avril 1932, Lebel est revenu sur l'essentiel des arguments de Whitton. Il a notamment contesté la dimension essentiellement nataliste de son projet, ne serait-ce que parce qu'il «faudrait être un peu naif pour croire qu'une allocation de 50 , qui ne couvrira qu'une faible partie des frais d'entretien d'un enfant, sera un stimulant exagéré ; que les parents se mettront sur les bras des douzaines d'enfants avec tous les ennuis et les sacrifices que leur éducation comporte, dans l'espoir de s'enrichir au moyen des allocations!» Il rappelait également que les allocations familiales ont eu une «influence à peine perceptible sur la natalité ». Voir Léon LEBEL, "Les allocations familiales », Le Devoir, 16 avril 1932.

71. Charlotte Whitton, citée dans CASQ, Troisième et Quatrième rapports. Les allocations familiales, $2^{\mathrm{e}}$ édition, Québec, ministère du Travail, p. 97. 
de reproduction) de chacun dans l'entreprise collective qu'est la société. En cela, si la montée des pratiques et des discours du risque et de l'assurance dans l'entre-deux-guerres constitue une nette indication du déplacement des enjeux de la question sociale dans le mode de régulation libérale, elle est toutefois bien insuffisante pour légitimer la reconnaissance politique d'un droit social. Cette reconnaissance, qui n'est également pas sans ambiguïtés, sera plutôt l'enjeu des luttes sociales et politiques de la seconde moitié du $\mathrm{XX}^{\mathrm{e}}$ siècle. 